\title{
Über den Pflegeprozess hinaus: Pflege mittels Routinedaten transparent und bewertbar machen
}

\author{
Dirk Hunstein
}

Online publiziert: 6. Dezember 2011

(C) Springer-Verlag 2011

Immer noch ist die Pflege eine „Black-Box“, wenn es um Fragen zum erforderlichen Aufwand und dem daraus folgenden Nutzen geht: Wie viel kostet (gute) Pflege? Was hier so ökonomisch-technisch klingt, lässt sich auch anders ausdrücken: Wie viel Pflege ist erforderlich, damit ein Patient sicher (und gut) versorgt wird?

Die Brisanz dieser Frage zeigt sich an dem missglückten Versuch, besonders aufwändige Pflege über die Komplexpauschale des PKMS finanzieren zu wollen. Es gibt Hinweise, dass teilweise weniger als $15 \%$ der tatsächlich hochaufwändigen Patienten vom PKMS erfasst werden, obwohl bei ihnen ein extrem hoher Pflegeaufwand (ABereich der PPR) von mehr als $200 \mathrm{~min}$ pro Tag anfällt (Hunstein [1]). Grund: Im PKMS wurden fiktive Patientenbzw. Pflegesituationen konstruiert, denen der empirische Beleg fehlt. Gleichzeitig wurde mit dem PKMS ein Leistungsanreizsystem geschaffen, in dem es vor allem darum geht, möglichst viele Leistungen entsprechend der formalen Vorgaben zu dokumentieren, anstatt sich damit zu beschäftigen, was der Patient in seiner individuellen Situation tatsächlich benötigt.

Bisher war es kaum möglich, Antworten auf die eingangs gestellten Fragen zu erhalten, weil die Daten dafür fehlten.

Mit der Verbreitung des elektronisch unterstützten Pflegeprozesses und geeigneter Assessmentverfahren (z. B. ergebnisorientiertes PflegeAssessment ePA-AC und LEP Nursing 3) stehen mittlerweile immer mehr Routinedaten zur Verfügung, die genutzt werden können, um diese, aber auch ganz alltägliche, Fragestellungen zu beantworten:
Wie lassen sich schon bei Eintritt des Patienten jene Patienten herausfiltern, die voraussichtlich einen besonders hohen Pflegeaufwand haben werden?

Wie lässt sich nachweisen, welche Erfolge Pflege bei bestimmten Patientengruppen erzielt hat (Stichwort: Qualitätsmessung mit Routinedaten)?

Welche Anhaltszahlen kann man nutzen, um den Personalbedarf in Abhängigkeit vom Pflegebedarf der Patienten abzuschätzen?

Wie kann man jene Patienten identifizieren, bei denen ein gutes Fallmanagement besonders wichtig ist, um die mittlere Verweildauer nicht zu überschreiten?

Wie könnte ein Alternativsystem zum PKMS aussehen, das die Pflegefachperson nicht mehr mit bürokratischer Zusatzdokumentation belastet und das statt der am grünen Tisch erdachten „Gründe“ die reale Patientensituation berücksichtigt?

\section{Literatur}

1. Hunstein D. Identifikation hochaufwändiger Patienten - Ein praxisnaher Lösungsansatz. Symposium des Deutschen Pflegerats „Pflege und DRG“. Berlin; 27. Mai 2011.

D. Hunstein $(\triangle)$

ePA Competence Center, Wiesbaden, Deutschland 